\title{
An assessment of the training and practice of circumcision by medical circumcisers in Ghana
}

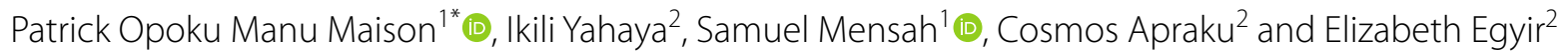

\begin{abstract}
Background: Circumcision is a common procedure in most parts of sub-Saharan Africa with an estimated prevalence of 95\% in Ghana. It has been documented that most circumcision related injuries in Ghana occur after circumcision by health care professionals who perform these circumcisions in hospitals and other healthcare facilities. In spite of the high rates of circumcision in Ghana, the expertise of circumcision providers and the safety of their practice has not been assessed. This study evaluates the training and practice of circumcision by health care professionals from across the country.
\end{abstract}

Methods: This is a questionnaire-based study of health care professionals who perform circumcision in Ghana. Data was extracted from pre-conference evaluation questionnaire completed by participants attending a circumcision training workshop from January to October 2019. These participants included responses on their training and practice of circumcision. Data was captured using the Epi-data software and analyzed with PASW Statistics for Windows, Version 18.0. Chicago: SPSS Inc.

Results: There were 378 participants seen over the study period who already performed circumcision. The majority of them were midwives 281 (74.3\%), followed by general nurses 89 (23.5\%). The duration of practice of circumcision ranged from 4 months to 23 years and one-third of them (33.3\%) had practiced circumcision for less than 1 year. The forceps guided technique was the most common (53.2\%) circumcision technique used followed by the dorsal slit technique (24.1\%). None of these participants who performed circumcision had any formal training on the procedure and all of them had experienced at least one adverse event such as excessive bleeding, insufficient skin coverage or redundant foreskin. Six (6) of them had seen patients who reported later with urethrocutaneous fistula and two of them had caused glans amputations. Only 102 (27\%) of these participants offered anesthesia for circumcision.

Conclusion: The majority of medical circumcisers in Ghana have no formal training for circumcision. They require formal circumcision training and certification to improve the practice of circumcision in Ghana.

Keywords: Circumcision, Health care professional, Training, Perform

\section{Background}

Circumcision is a common procedure in most parts of sub-Saharan Africa. The World Health Organization (WHO) estimates about 90\% prevalence of circumcision

\footnotetext{
*Correspondence: pmaison@uccsms.edu.gh

1 Department of Surgery, School of Medical Sciences, College of Health

and Allied Sciences, University of Cape Coast, Cape Coast, Ghana

Full list of author information is available at the end of the article
}

in West Africa and 95\% prevalence in Ghana [1]. Circumcision is usually performed for cultural or religious reasons in Ghana. Almost all circumcisions in Ghana are performed in the neonatal period or in infancy [2]. Circumcision was predominantly performed by traditional circumcisers but most circumcisions in the country are now performed by health care professionals [2].

Although, it has been shown that circumcision complications are rare when conducted by trained and 
experienced providers, Appiah et al. documented that most circumcision related injuries in Ghana resulted after circumcision by health care professionals who perform these circumcisions in hospitals and other healthcare facilities [1,3]. In fact, in a population based study in rural Ghana, Gyan et al. observed that comparatively, hygiene practices such as hand washing, cleaning of the penile area and cleaning of instruments were poorer among formal healthcare professionals than informal providers [4]. Most of these health professionals are general nurses, midwives, physician assistants and doctors who often, have not had any formal training on circumcision.

In spite of the high rates of circumcision in Ghana, the expertise of circumcision providers and the safety of their practice has yet to be assessed. There are various kinds of health care professionals performing circumcision in the health facilities without proper training, resulting in serious circumcision related tragedies such as penile amputations, urethrocutaneous fistulae, glans or penile ischemic necrosis, etc. [3].

This study evaluates the training and practice of circumcision by a section of health care professionals from across the country.

\section{Methods}

This cross-sectional study was conducted from January to October 2019. Health care professionals who perform circumcision in Ghana were asked to complete a questionnaire after obtaining informed consent. These health personnel were among participants from both private and public health facilities across the country attending a circumcision training workshop organized by a team led by the lead author. The circumcision workshop was to train all categories of health personnel who either already performed circumcision or wanted training on circumcision. The curriculum of the workshop had been approved by the Medical and Dental Council of Ghana for a continuous professional education program. The study data was extracted from the pre-conference evaluation questionnaire administered to all participants for the workshop. Participants who already performed circumcision included responses on their training for circumcision, circumcision techniques used, administration of anesthesia for circumcision and complications of circumcision they had experienced. The workshop was repeated monthly in each of the 10 regional capitals of Ghana for health personnel who work in that region.

Data was captured using the Epi-data software and analyzed with PASW Statistics for Windows, Version 18.0. Chicago: SPSS Inc.

\section{Results}

There were 378 participants who already performed circumcision out of a total of 1952 participants who attended the training workshops over the study period. The 378 participants included healthcare assistants, general nurses, midwives, nurse anesthetists, physician assistants and doctors. The majority of them were midwives $281(74.3 \%)$, followed by general nurses $89(23.5 \%)$ as shown in Table 1. The duration of practice of circumcision ranged from 4 months to 23 years and as shown in Fig. 1, one-third of them (33.3\%) had practiced circumcision for less than 1 year. The forceps guided technique, also known as the amputation or free hand technique was the most common (53.2\%) circumcision technique used followed by the dorsal slit technique (24.1\%). None of these participants who performed circumcision had any formal training on the procedure and all of them complained of at least one adverse event that accompanies the procedure when they perform it. All of them had encountered early complications such as excessive bleeding, insufficient skin coverage or redundant foreskin. Six (6) of them had seen patients who reported later with urethrocutaneous fistula and two of them had caused glans amputations. Only 102 (27\%) of these participants offered anesthesia for circumcision.

\section{Discussion}

Infant male circumcision is commonly practiced in many parts of the world, by both formal health service providers such as doctors, medical assistants, midwives, general nurses and informal health service providers including traditional circumcision providers, traditional birth attendants, hospital workers with no formal medical training, e.g., orderlies and attendants, religious leaders and traditional medicine men or women [5]. As a surgical procedure, it is expected that male circumcision be performed to the required standard by all circumcision service providers with the requisite training. Yet, health service providers often do not receive any formal training and education to perform circumcision to required standards. Studies show that complications following circumcision are usually due to the lack of structured training on circumcision for medical circumcisers. Hence, these studies recommend structured and formal training to reduce complications associated with infant male circumcision $[5,6]$. In this study, none of the 378 participants who performed circumcision had received any formal training on the procedure despite the fact that two-thirds of them had performed circumcision for more than 1 year. They had all learnt to perform circumcision by observing other colleagues who performed the procedure. At least each of them had encountered early complications such as excessive bleeding, insufficient 
Table 1 Distribution of health care professionals and circumcision techniques used

\begin{tabular}{ll}
\hline Category & Number (\%) \\
\hline Category of health care professionals who perform circumcision & $5(1.3 \%)$ \\
Healthcare assistant & $89(23.5 \%)$ \\
General nurse & $281(74.3 \%)$ \\
Midwife & $2(0.6 \%)$ \\
Nurse anesthetist & $1(0.3 \%)$ \\
Medical doctor & $378(100 \%)$ \\
Total & Number (\%) \\
\hline Technique & \\
\hline Circumcision technique used & $6(1.6 \%)$ \\
Gomco clamp only & $39(10.3 \%)$ \\
Plastibel only & $91(24.1 \%)$ \\
Dorsal slit only & $201(53.2 \%)$ \\
Amputation only & $9(2.4 \%)$ \\
Dorsal slit+Gomco clamp/Plastibel & $11(2.9 \%)$ \\
Amputation technique+ Gomco clamp/Plastibel & $5(1.3 \%)$ \\
Gomco clamp+Plastibel & $16(4.2 \%)$ \\
Dorsal slit+Amputation technique & $378(100 \%)$ \\
Total &
\end{tabular}

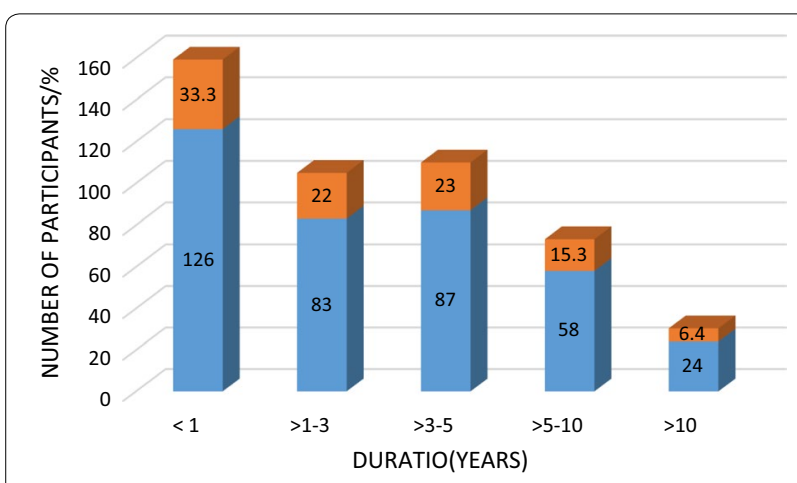

Fig. 1 Number of participants and duration of experience

skin coverage or redundant foreskin. Six (6) of them had seen patients who developed urethrocutaneous fistula post circumcision. It is possible that there are other late complications including urethrocutaneous fistulae, penile skin bridges, epidermal inclusion cysts and even glans or penile amputations that may not have reported to them. This is because most parents report late complications of circumcision to urologists and not to the circumciser as they do not attribute the complication to the circumcision procedure due to the long period it takes for the complications to manifest [3, 7]. In Uganda, it was shown that even trained doctors needed to complete 100 circumcisions to significantly reduce the incidence of adverse events of circumcision [8]. This therefore indicates that untrained medical personnel such as midwives, nurses, etc. are likely to have many adverse events from the procedure as was observed in this study.

The World Health Organization recommends anesthesia for neonatal circumcision $[9,10]$. Local anesthesia using a surface cream like eutectic mixture of local anesthetics (EMLA), or using 1\% lidocaine for targeted or ring block at the base of the penis helps reduce pain of circumcision for the patient and also makes him more cooperative for the procedure to be done, reducing risk of iatrogenic injuries and complications. In this study, only $27.0 \%$ of participants performed circumcision under anesthesia. This low rate of anesthesia for circumcision is despite the fact that local anesthetic agents like Lidocaine are readily available and affordable. This low rate may be due to poor comprehension of the principles of circumcision, which would have been acquired through formal training.

In infants and neonates, it is safest to use techniques such as the device methods with Plastibel or Gomco clamp which protect the glans penis or use surgical techniques like the dorsal slit or sleeve technique where excision of foreskin is done with the glans penis in view to reduce the risk of injury to it [11]. In the study by Appiah et al. on circumcision related tragedies in Ghana, almost $7 \%$ (5 out of 72 ) of the complications were penile amputations, mostly due to forceps guided/amputation technique [3]. In this study, the forceps guided technique/ amputation technique was the most common technique 
used despite the fact that in neonates and infants, this technique risks injuring the glans or the phallus due to its relatively small size. This is consistent with the findings of Gyan et al. who observed that $88.9 \%$ of circumcisions performed in rural Ghana were done with the forceps guided technique [4]. The principles of circumcision recommend excision of foreskin while the glans penis is under direct vision as this helps to reduce risk of glans or penile injury [9]. Formal training may have exposed these participants to some of these guiding principles or recommendations and may have reduced the popularity of the forceps guided technique where foreskin is excised without the glans penis in view.

\section{Conclusion}

The majority of medical circumcisers in Ghana have no formal training for circumcision. Formal circumcision training and certification will reduce circumcision risk and improve the practice of circumcision in Ghana. A study of the practice of participants to the circumcision training workshop should be conducted in the future to determine the effects of formal training on their practice.

\section{Abbreviations}

WHO: World Health Organization; SPSS: statistical package for the social sciences; PASW: predictive analytics software; EMLA: eutetic mixture of local anesthetics.

\section{Acknowledgements \\ Not applicable.}

\section{Authors' contributions}

POMM: Conceived and designed the study and collected the data. Drafted the article with inputs from IY and SM. IY: Design of the work, data collection and drafting the article. Critically revised the draft. SM: Data analysis with inputs from CA and drafting the article. Critically revised the draft. CA: Acquisition of data and data analysis. Critically revised the draft. EE: The acquisition of data. Substantively revised the draft. All authors read and approved the final manuscript.

\section{Funding}

The study received no external funding.

\section{Availability of Data and Materials}

The datasets used and/or analyzed during the current study are available from the corresponding author on reasonable request.

\section{Ethics approval and consent to participate}

Data for this research was extracted from the pre-conference evaluation questionnaire that was administered to participants who were attending a circumcision training workshop. The curriculum of the workshop, all the conference evaluation questionnaires and the study were approved by the Medical and Dental Council of Ghana after academic and ethical considerations were evaluated. The Council is however not an IRB committee and hence does not give reference numbers. This study did not involve patients and no biological samples were taken. The study participants were comfortable and provided written consent by signing the declarations on the consent form. Participation was voluntary.

\section{Consent for publication}

A written informed consent was taken from all participants included in this research to publish the data contained within this study.

\section{Competing interests}

Not applicable.

\section{Author details}

${ }^{1}$ Department of Surgery, School of Medical Sciences, College of Health and Allied Sciences, University of Cape Coast, Cape Coast, Ghana. ${ }^{2}$ Holy Family Hospital, Techiman, Ghana.

Received: 21 April 2020 Accepted: 10 June 2020

Published online: 31 August 2020

\section{References}

1. Weiss H, Larke N, Halperin D, Schenker I (2010) Neonatal and child male circumcision: a global review. UNAIDS Technical Bulletin. UNAIDS, Geneva

2. Report on neonatal, infant and pre-pubertal male circumcision practices in traditional and clinical settings in Ghana: World Health Organisation (2008)

3. Appiah AA et al (2016) Circumcision-related tragedies seen in children at the Komfo Anokye Teaching Hospital, Kumasi, Ghana. BMC Urol 16:65. https://doi.org/10.1186/s12894-016-0183-1

4. Gyan T et al (2017) Determinants of morbidity associated with infant male circumcision: community-level population-based study in rural Ghana. Trop Med Int Health 22(3):312-322. https://doi.org/10.1111/ tmi.12829

5. Weiss HA, Larke N, Halperin D, Schenker I (2010) Complications of circumcision in male neonates, infants and children: a systematic review. BMC Urol 10(1):2

6. Ekenze SO, Ezomike UO (2013) Complications of neonatal circumcision requiring surgical intervention in a developing country. J Trop Pediatr 59(4):292-297

7. Yegane R-A, Kheirollahi A-R, Salehi N-A, Bashashati M, Khoshdel J-A, Ahmadi M (2006) Late complications of circumcision in Iran. Pediatr Surg Int 22:442-445

8. Kiggundu V, Watya S, Kigozi G, Serwadda D, Nalugoda F, Buwembo D et al (2009) The number of procedures required to achieve optimal competency with male circumcision: findings from a randomized trial in Rakai, Uganda. BJU Int 104(4):529-532

9. Simpson E, Carstensen J, Murphy P (2014) Neonatal circumcision: new recommendations \& implications for practice. Mo Med 111(3):222-230

10. World Health, Organization Jhpiego (2011) Manual for early infant male circumcision under local anaesthesia. WHO, Geneva

11. Weiss H (2008) Organization WH, HIV/AIDS JUNPo. Male circumcision: global trends and determinants of prevalence, safety, and acceptability. World Health Organization, Geneva

\section{Publisher's Note}

Springer Nature remains neutral with regard to jurisdictional claims in published maps and institutional affiliations. 